\title{
THE RELATION BETWEEN TWO SYMMETRIC INTEGRALS
}

\section{GEORGE CROSS}

1. Introduction. This paper is concerned mainly with the $S C P$ integral of Burkill [3] and the $P^{2}$-integral of James $[6 ; 7]$; for an explanation of the notation and terminology used here the reader is referred to $[1 ; 2 ; 3 ; 6$ and 7$]$.

It is known that the $8^{2}$-integral of James [7] includes the $C_{1} P$ integral of Burkill [1]. Both of these integrals are of Perron type and were developed using major and minor functions defined by means of unsymmetric derivates. It is convenient to refer to these integrals as "unsymmetric" integrals. For the solution of problems in trigonometric series it is more profitable to work with "symmetric" integrals, i.e., integrals defined by means of symmetric derivates. Symmetric integrals have been defined by Burkill [3] and by James [7] and because of the similarity of construction of the two integrals, and the similarity of the results obtained by means of these integrals, it is reasonable to expect that a simple relationship holds between them. It is proved here that the $P^{2}$-integral includes the $S C P$-integral, and a special relation that exists between the integrals when the integrand is periodic is demonstrated. It is further shown that $P^{2}$-integrability on an interval does not imply $S C P$-integrability there, a result which perhaps is unexpected since $8^{2}$-integrability is known to imply $C P$ integrability on every closed subinterval [7].

2. Theorems and an example. In this section the notation of [6] and [7] are combined but this should cause no confusion since, by the remark of James, $\$ 7$ of [8], the $P^{2 m}$-integral for $m=1$ is the same as the original $P^{2}$-integral.

THEOREM I. If $f(x)$ has an SCP-integral in $(a, b)$ with basis $B$, then $f(x)$ is $P^{2}$-integrable over $\left(a_{i} ; x\right)$ where $\left(a_{i}\right) \equiv\left(a_{1}, a_{2}\right)=(a, b)$. Furthermore, if

$$
F_{1}(x)=(S C P, B) \int_{a}^{x} f(t) d t
$$

then $F_{1}(x)$ is Denjoy integrable, and if

$$
F(x)=(D) \int_{a}^{x} F_{1}(t) d t,
$$

Received by the editors December 22, 1961. 
then, for $a \leqq x \leqq b$,

$$
-\int_{\left(a_{i}\right)}^{x} f(x) d_{2} x=F(x)-\sum_{1}^{2} \lambda\left(x ; a_{i}\right) F\left(a_{i}\right),
$$

where

$$
\lambda\left(x ; a_{i}\right)=\prod_{i \neq j}\left(\frac{x-a_{j}}{a_{i}-a_{j}}\right) .
$$

Proof. Let $M(x)$ be any $S C P$-major function of $f(x)$ with basis $B$ and let

$$
G(x)=(D) \int_{a}^{x} M(t) d t
$$

Then $G(x)$ is continuous $[9$, p. 201 and p. 250] and

$$
G(x+h)+G(x-h)-2 G(x)=(D) \int_{x}^{x+h} M(t) d t-(D) \int_{x-h}^{x} M(t) d t
$$

This gives

$$
\begin{aligned}
\partial^{2} G(x) & \equiv \liminf _{h \rightarrow 0} h^{-2}\{G(x+h)+G(x-h)-2 G(x)\} \\
& =\liminf _{h \rightarrow 0} h^{-1}\left\{(1 / h)(D) \int_{x}^{x+h} M(t) d t-(1 / h)(D) \int_{x-h}^{x} M(t) d t\right\} \\
& =S C D_{*} M(x) .
\end{aligned}
$$

It follows that

$$
\partial^{2} G(x) \geqq f(x)
$$

and

$$
\partial^{2} G(x)>-\infty,
$$

except possibly on a countable set. This shows that the function

$$
Q(x) \equiv G(x)-\sum_{1}^{2} \lambda\left(x ; a_{i}\right) G\left(a_{i}\right)
$$

is a $P^{2}$-major function of $f(x)$.

An analogous construction yields a $P^{2}$-minor function $q(x)$ defined by

$$
q(x) \equiv g(x)-\sum_{1}^{2} \lambda\left(x ; a_{i}\right) g\left(a_{i}\right)
$$


where

$$
g(x)=(D) \int_{a}^{x} m(t) d t
$$

and $m(x)$ is an $S C P$-minor function of $f(x)$ with basis $B$. Now for $\epsilon>0$, and $t \in B$, it is true that $0 \leqq M(t)-m(t) \leqq M(b)-m(b)<\epsilon$. The function $M(x)-m(x)$ is thus Lebesgue integrable on $[a, b]$ and the $P^{2}$-integrability of $f(x)$ follows from the fact that

$$
\begin{aligned}
0 & \leqq q(c)-Q(c)=\int_{a}^{c}[m(t)-M(t)] d t+\frac{c-a}{b-a} \int_{a}^{b}[M(t)-m(t)] d t \\
& <\frac{c-a}{b-a} \int_{a}^{b}[M(t)-m(t)] d t<\epsilon(c-a) .
\end{aligned}
$$

Since $f(x)$ is $S C P$-integrable, with basis $B$, there is a sequence $\left\{M_{n}(x)\right\}$ of $S C P$-major functions such that

$$
0 \leqq M_{n}(x)-F_{1}(x)<1 / n, \quad x \in B, n=1,2,3, \cdots .
$$

Each $M_{n}(x)-F_{1}(x)$ is thus Lebesgue integrable, and the Denjoy integrability of $F_{1}(x)$ follows since $M_{n}(x)$ is Denjoy integrable.

Corresponding to the sequence $\left\{M_{n}(x)\right\}$ in (2) there is a sequence $\left\{G_{n}(x)\right\}$ such that

$$
\begin{aligned}
0 \leqq G_{n}(x)-F(x) & \equiv D \int_{a}^{x}\left[M_{n}(t)-F_{1}(t)\right] d t \\
& \equiv D \int_{a}^{x} f(t) d t
\end{aligned}
$$

where $f(t)=M_{n}(t)-F_{1}(t), t \in B$ and $f(t)=0, t \notin B$. It follows that

$$
0 \leqq G_{n}(x)-F(x)<\frac{x-a}{n} \leqq \frac{b-a}{n},
$$

for all $x$, and so

$$
G_{n}(x) \rightarrow F(x)
$$

for all $x$.

The corresponding $P^{2}$-major function $Q_{n}(x)$ tends to the righthand side of (1), and by the definition of the $P^{2}$-integral, the sequence $\left\{Q_{n}(x)\right\}$ tends to the left-hand side of (1). This completes the proof of Theorem I. 
The relationship between a definite $S C P$-integral and a definite $P^{2}$-integral when the integrand is periodic takes a particularly simple form and is analogous to the relation between the definite $C_{1} P$ integral and the definite $\boldsymbol{8}^{2}$-integral under similar circumstances (cf. [4]). The result may be stated as follows:

TheOREM II. Let $f(x)$ be SCP-integrable over $[-b, b]$ with basis $B$, and periodic with period $2 b$. Let $\left(a_{i}\right) \equiv(-2 b, 2 b)$. Then

$$
\frac{1}{b} \int_{\left(a_{i}\right)}^{0} f(t) d_{2} t=(S C P, B) \int_{-b}^{b} f(t) d t .
$$

Proof. Let

$$
\begin{array}{ll}
F_{1}(x)=(S C P, B) \int_{-b}^{x} f(t) d t, & x \in B \\
F(x)=(D) \int_{-b}^{x} F_{1}(t) d t . &
\end{array}
$$

Then

$$
\begin{aligned}
F_{1}(x) & =(S C P, B) \int_{-b}^{x} f(t) d t \\
& =\frac{x}{2 b}(S C P, B) \int_{-b}^{b} f(t) d t+G(x) \\
& \equiv x \mu+G(x), \quad x \in B,
\end{aligned}
$$

where $G(x)$ is Denjoy integrable and periodic with period $2 b$ in its domain of definition. It follows that $F(x)$ may be written as $G_{1}(x)$ $+G_{2}(x)$ where $G_{1}(x)=\mu x^{2} / 2$ and $G_{2}(x)$ is periodic with period $2 b$. By Theorem $I$ it is clear that

$$
-\int_{\left(a_{i}\right)}^{0} f(t) d_{2} t=F(0)-\sum_{1}^{2} \lambda\left(0 ; a_{i}\right) F\left(a_{i}\right) .
$$

The right-hand side of (3) reduces to $-2 \mu b^{2}$ by an easy calculation and this gives the desired equality.

Although the $C_{r} P$-integral does not include the $p^{r+1}$-integral, James has proved [7] that $\boldsymbol{\rho}^{r+1}$-integrability over $\left(a_{i} ; x\right)$ implies $C_{r} P$-integrability over any closed sub-interval of $\left(a_{1}, a_{r+1}\right)$. On the other hand there are functions which are $P^{2}$-integrable and not $C P$ integrable [6], indeed functions which are $P^{2}$-integrable and not $C_{r} P$-integrable for any $r[5]$. The following theorem and example 
show that there are functions which are $P^{2}$-integrable and not $S C P$ integrable.

Theorem III. If $f(x)$ is $P^{2}$-integrable over $\left(a_{i} ; x\right), F(x)$ $\equiv-\int_{a_{i}}^{x} f(t) d t$, if $f(x)$ is SCP-integrable over $(a, b)$ and if $G(x)$ $=(S C P, B) \int_{a_{i}}^{x} f(x) d x$, then $F^{\prime}(x)$ exists for all $x$ in $B$ and

$$
F^{\prime}(x)=G(x)-\frac{1}{b-a} \int_{a}^{b} G(t) d t, \quad x \in B .
$$

Proof. It follows from (1) that

$$
\frac{F(x+h)-F(x)}{h}=\frac{1}{h} \int_{0}^{h} G(x+t) d t-\frac{1}{b-a} \int_{a}^{b} G(t) d t,
$$

and the result is a consequence of the $C$-continuity of $G(x)$ for $x \in B$ [3].

The final object of this paper is to display a function which is $P^{2}$ integrable over $[a, b]$ and not $S C P$-integrable (with any basis) over every subinterval $[c, d], a \leqq c<d \leqq b$. Let

$$
\begin{aligned}
& F(x)=\left\{\begin{array}{cc}
x \cos \frac{1}{x}, & x \neq 0 \\
0, & x=0
\end{array}\right. \\
& f(x)=\left\{\begin{array}{cc}
\frac{-\cos 1 / x}{x^{3}}, & x \neq 0 \\
0, & x=0 .
\end{array}\right.
\end{aligned}
$$

It is known [6] that $f(x)$ is $P^{2}$-integrable over any interval that includes the origin and

$$
F(x)=-\int_{\left(a_{i}\right)}^{x} f(x) d x+\left(\frac{b-x}{b-a}\right) F(a)+\left(\frac{x-a}{b-a}\right) F(b),
$$

while $F^{\prime}(0)$ does not exist.

If $f(x)$ were $S C P$-integrable over $[0, d], 0<d \leqq b$ with basis $B$, then by Theorem III, $F^{\prime}(x)$ would exist at $x=0$.

\section{REFERENCES}

1. J. C. Burkill, The Cesdro-Perron integral, Proc. London Math. Soc. (2) 34 (1932), 314-322.

2. The Cesdro-Perron scale of integration, Proc. London Math. Soc. (2) 39 (1935), 541-552.

3. - Integrals and trigonometric series, Proc. London Math. Soc. (3) 1 (1951), 46-57. 
4. G. E. Cross, The relation between two definite integrals, Proc. Amer. Math. Soc. 11 (1960), 578-579.

5. H. W. Ellis, On the relation between the $P^{2}$-integral and the Cesdro-Perron scale of integrals, Trans. Royal Soc. Canada III 46 (1952), 29-32.

6. R. D. James, $A$ generalized integral. II, Canad. J. Math. 2 (1950), 297-306.

7. - Generalized nth primitives, Trans. Amer. Math. Soc. 76 (1954), 149176.

8. - Summable trigonometric series, Pacific J. Math. 6 (1956), 99-110.

9. S. Saks, Theory of the integral, Warsaw, 1937.

The UNIVERSiTy OF Western ONTARIo 